\title{
Direct Quantitation of Active Ingredients in Solid Artesunate Antimalarials by Noncovalent Complex Forming Reactive Desorption Electrospray Ionization Mass Spectrometry
}

\author{
Leonard Nyadong, ${ }^{\mathrm{a}}$ Sameer Late, ${ }^{\mathrm{b}}$ Michael D. Green, ${ }^{\mathrm{c}}$ Ajay Banga, ${ }^{\mathrm{b}}$ and \\ Facundo M. Fernández ${ }^{a}$ \\ a School of Chemistry and Biochemistry, Georgia Institute of Technology, Atlanta, Georgia, USA \\ ${ }^{b}$ Department of Pharmaceutical Sciences, College of Pharmacy and Health Sciences, Mercer University, \\ Atlanta, Georgia, USA \\ ${ }^{c}$ Division of Parasitic Diseases, National Center for Infectious Diseases, Centers for Disease Control and \\ Prevention, Atlanta, Georgia, USA
}

The direct quantitation of active ingredients in solid pharmaceutical tablets by desorption electrospray ionization mass spectrometry (DESI MS) is complicated by the dependence of the DESI signal on variables such as spray angles and distances, morphological sample properties, and the difficulty of properly incorporating an internal standard. Here, a DESI MS method for the direct quantitative screening of widely counterfeited antimalarial tablets containing artesunate is presented. This method is based on reactive DESI, where analyte desorption and ionization occur by the formation of noncovalent complexes between alkylamine molecules in the DESI spray solution and artesunate molecules exposed on the sample surface in the open air. For quantitation purposes, the internal standard $d_{4}$-artesunic acid was synthesized by esterification of $\mathrm{d}_{4}$-succinic anhydride and dihydroartemisinin, and homogeneously dispersed on the tablet surface via a controlled deposition procedure. The analyte-to-internal standard signal intensity ratio was observed to be largely independent of all DESI variables, only showing dependence on tablet hardness. Analysis of artesunate tablet standards prepared with known amounts of the active ingredient in the 0.02 to $0.32 \mathrm{mg}$ artesunate $\mathrm{mg}^{-1}$ tablet range resulted in a calibration curve with good linearity $(r=0.9985)$. Application of this method to the direct quantitation of genuine artesunate tablets from Vietnam showed a $6 \%$ $(n=4)$ precision and $94 \%$ accuracy after the spectral data were corrected for tablet hardness. (J Am Soc Mass Spectrom 2008, 19, 380-388) (c) 2008 American Society for Mass Spectrometry

$\mathrm{T}$ The development of methodologies that allow the quantitative determination of physiologically active analytes in complex matrices is an ongoing trend in analytical and biological mass spectrometry. In this sense, high-performance liquid chromatography (LC) coupled to mass spectrometry (MS) via electrospray ionization (ESI), or atmospheric pressure chemical ionization (APCI), has become the de facto routine quantitative analytical tool in clinical chemistry, drug discovery, and proteomics [1-3]. Although LC-MS is characterized by one of the highest peak capacities of contemporary analytical separation methodologies [4], alternative techniques are constantly being devel-

Address reprint requests to Dr. Facundo M. Fernández, School of Chemistry and Biochemistry, Georgia Institute of Technology, 901 Atlantic Drive NW, Atlanta, GA 30332-0400, USA. E-mail: facundo.fernandez@chemistry. gatech.edu oped to improve sample throughput, eliminate crosscontamination, and increase reproducibility.

The emergence of ambient desorption ionization techniques such as desorption electrospray ionization (DESI) [5], direct analysis in real time (DART) [6], and others has enabled the direct sampling of complex solid materials in the open air with minimal or no sample preparation. DESI makes use of a pneumatically assisted electrospray jet, which is directed unto the sample surface to desorb the condensed-phase analyte(s). Following desorption, the analyte-containing charged droplets scattered off the sample surface are sampled by a mass spectrometer. DESI enables the analysis of materials over a large mass range and provides the capability to perform ion-molecule reactions at the interface between the charged microdroplets and the condensed-phase analytes, resulting in improved detection selectivity and sensitivity [7]. These reactions are achieved by the 
introduction of reagents into the DESI spray solution in what is now generally referred to as "reactive" DESI [7-9].

One very promising application of DESI is the direct screening of solid pharmaceutical formulations for drug quality control. The majority of DESI work done in this area thus far has been qualitative [7, 10-16]. The large dependence of the DESI signal on various DESI parameters such as spray tip-to-surface distance, sample-tospectrometer orifice distance, spray incident angle, and collection angle might be one of the major contributing factors limiting progress in this direction. Fixed-geometry DESI probes have been shown to somewhat mitigate this problem [17]. However, in the case of pharmaceutical solid dosage forms there is an additional dependence of the DESI signal on sample properties such as hardness and sample shape, the latter influencing incidence and collection angles. In their seminal work, Cooks and coworkers first suggested that the use of an internal standard (IS) should enable quantitative applications, and demonstrated the feasibility of using DESI in the quantitative analysis of phenylalanine deposited unto glass substrates [18]. DESI has also been applied to the quantitative analysis of alkaloids separated by thinlayer chromatography [19], but to the best of our knowledge, there have been no reports where DESI is employed for the direct quantitation of active pharmaceutical ingredients in solid tablets.

One major challenge faced today by public health authorities and the pharmaceutical industry relates to the counterfeiting of various drug formulations [20]. Due to its high cost and demand, one pharmaceutical that has been highly counterfeited is the oral antimalarial artesunate [21]. Artemisinin-derived antimalarial drugs such as artemether, arteether, dihydroartemisinin, and artesunate, as mono-therapies, or in combination with co-drugs such as lumefantine and mefloquine are currently the only effective treatment for multidrug resistant Plasmodium falciparum malaria [21] in many parts of Asia and Africa. Recently discovered fake artesunate samples have been shown to contain small subtherapeutic quantities of the correct active ingredient, possibly introduced to defeat current colorimetric authentication tests in use in the field [7,22]. This is one of the most dangerous types of fake medicines for public health, as their widespread distribution could favor the emergence of drug resistant parasite strains. Hence, there is urgency for the development of rapid screening methods to quantitatively assess the quality of antimalarial tablets collected in the field.

Here, we exploit our previously reported qualitative reactive DESI approach [7, 12] for the direct quantitative analysis of artesunate tablets. Due to the challenges posed by the incorporation of a controlled, well-dispersed amount of IS into a solid tablet, several deposition methods are explored and compared for the development of a quantitative analytical tool for the direct sampling of solid antimalarial formulations. We show that the large dependence of the DESI signal intensity with various experimental variables and sample properties can be overcome by the homogeneous deposition and cocrystallization of a deuterated IS on the tablet surface. The method proposed here should also be applicable to the screening of other solid dosage forms.

\section{Experimental}

\section{Samples and Reagents}

All reagents were used as received. HPLC-grade acetonitrile (Fisher, Hampton, NH) and dodecylamine (DDA; Sigma-Aldrich, St. Louis, MO) were used for DESI and reactive DESI experiments. Ultrapure water (18.2 $\mathrm{M} \Omega \mathrm{cm}^{-1}$ ) was obtained from a Nanopure purification unit (Barnstead, San Jose, CA). Genuine artesunate tablets manufactured by Mekophar Chemical Pharmaceutical Joint-Stock Co. (Ho Chi Minh City, Vietnam), stated to contain $50 \mathrm{mg}$ of artesunate per tablet with an average total tablet weight of $320 \mathrm{mg}$, were used for all characterization and optimization studies. The amount of artesunate in these samples was also measured by liquid chromatography with spectrophotometric detection, following the procedure described previously [22]. Dihydroartemisinin (AApin Chemicals Ltd., Abingdon, UK), $\mathrm{d}_{4}$-succinic anhydride, triethylamine, tetrahydrofuran, acetic acid, anhydrous sodium sulfate, ethylacetate, and hexane (Sigma-Aldrich, St. Louis, MO) were used in the synthesis of $\mathrm{d}_{4}$-artesunic acid. Cab-o-sil, talc, magnesium stearate (Acros Organics, Morris Plains, NJ), microcrystalline cellulose (Mallinckrodt Baker Inc., Phillipsburg, NJ), croscarmellose sodium, and spray-dried lactose (FMC Corp., Newark, DE) were used as excipients for the preparation of artesunate tablet standards. Artesunic acid (Structure 1) was purchased from AApin Chemicals Ltd. All genuine and standard artesunate tablet samples were stored at $4{ }^{\circ} \mathrm{C}$ until analyzed.

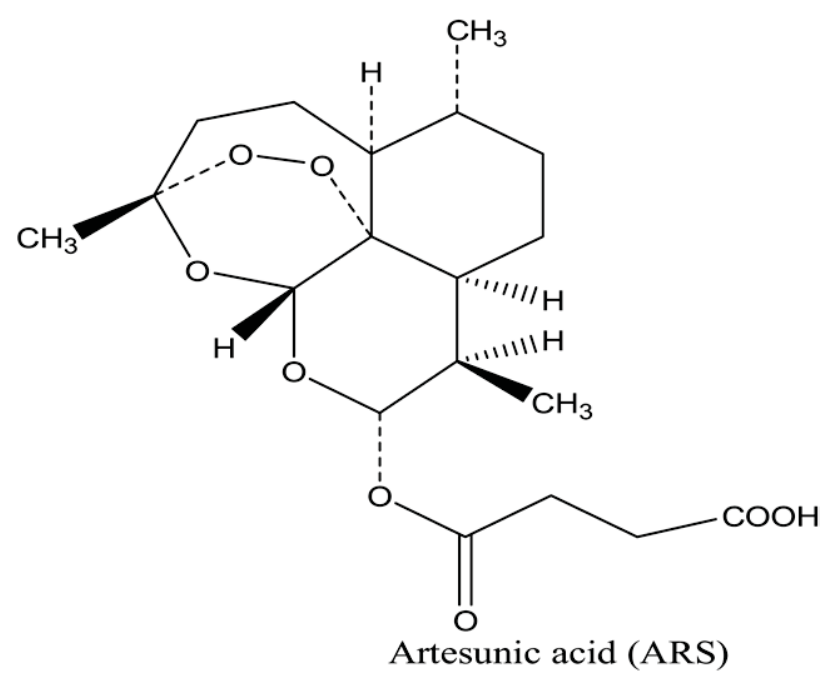

Structure 1. 


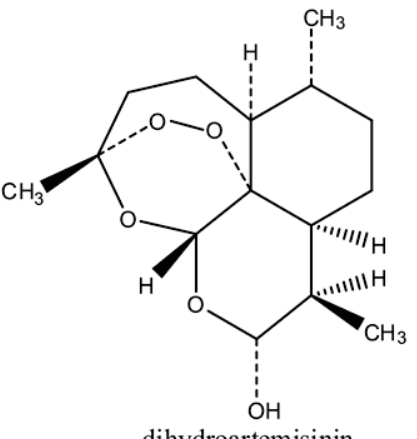

dihydroartemisinin

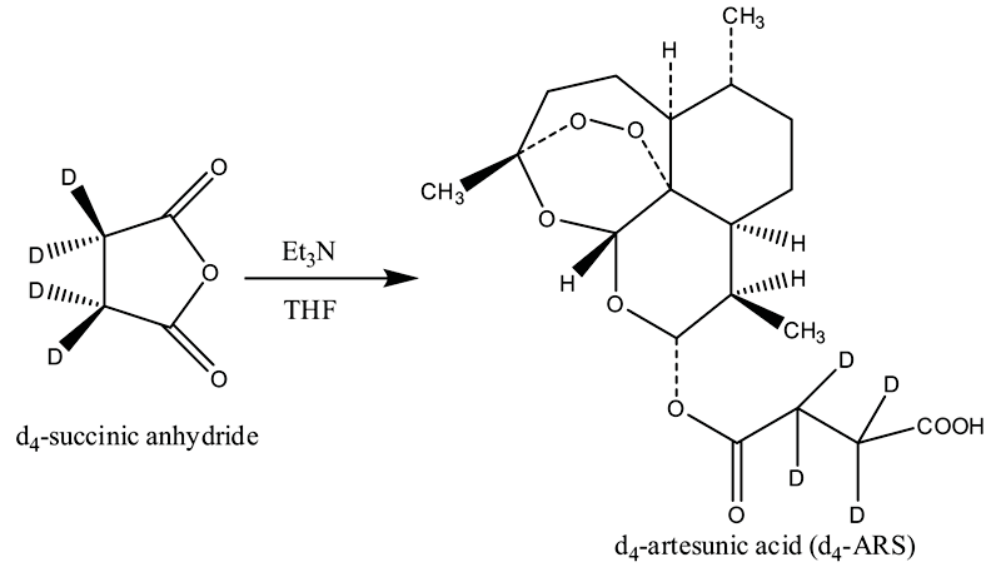

Scheme 1.

\section{Synthesis of Internal Standard}

The IS, $\mathrm{d}_{4}$-artesunic acid, was synthesized by the base catalyzed esterification of dihydroartemisinin with $d_{4}{ }^{-}$ succinic anhydride according to Scheme 1 . A previously reported procedure for the synthesis of artesunic acid $[23,24]$ was followed with some modifications, using dihydroartemisinin as the starting material. In a typical synthetic procedure, $250 \mathrm{mg}$ of dihydroartemisinin was stirred for $2 \mathrm{~min}$ in $5 \mathrm{~mL}$ of tetrahydrofuran (THF); 125 $\mathrm{mg}$ of $\mathrm{d}_{4}$-succinic anhydride and $500 \mu \mathrm{L}$ of triethylamine were then added to the reaction flask and stirred for $3 \mathrm{~h}$ at room temperature. At the end of the reaction, $20 \mathrm{~mL}$ of cold water, previously adjusted to $\mathrm{pH} 4.0$ with dilute acetic acid, was added to the mixture, and the $\mathrm{pH}$ finally adjusted to 4.0 by addition of dilute acetic acid. Extraction of the reaction product was then performed on ice using three separate 20-mL portions of 60:40 ethylacetate:hexane. The extract was washed with three 10-mL portions of chilled water previously adjusted to $\mathrm{pH} 4.0$ and then dried over anhydrous sodium sulfate. The solvent was then evaporated in a Rotavap $\left(30^{\circ} \mathrm{C}\right)$ to an estimated final volume of $5 \mathrm{~mL}$, after which it was changed by three separate additions and evaporations of $10-\mathrm{mL}$ portions of hexane. The liquid volume was reduced to $3 \mathrm{~mL}$ and left to evaporate to dryness in a hood at ambient temperatures. The crude product was purified by recrystallization in hexane. Purity was assessed by electrospray ionization MS of a $50: 50 \mathrm{CH}_{3} \mathrm{CN}$ : $\mathrm{H}_{2} \mathrm{O}$ solution containing $1 \mu \mathrm{M}$ of the product in $100 \mu \mathrm{M}$ DDA. The IS ESI spectrum showed predominantly a single peak at $\mathrm{m} / \mathrm{z} 574$ corresponding to $\left[\mathrm{d}_{4}-\mathrm{ARS}+\right.$ $\mathrm{DDA}+\mathrm{H}]^{+}$, indicating a high-purity reaction product, with the expected 4 mass unit shift relative to the artesunate standard noncovalent complex [ARS + DDA $+\mathrm{H}]^{+}$.

\section{Preparation of Artesunate Tablet Standards}

Artesunate tablet standards were composed of three major constituents: excipient mixture A, spray dried lactose, and artesunic acid. Excipient mixture A was composed of $69 \%$ microcrystalline cellulose, $1 \%$ fumed silicon dioxide (cab-o-sil), 5\% talc, 20\% croscarmellose sodium, and $5 \%$ magnesium stearate. This mixture was prepared as follows: microcrystalline cellulose, cab-osil, and croscarmellose sodium were passed through a 20 mesh sieve and mixed together in a zip-lock bag for $5 \mathrm{~min}$. Talc and magnesium stearate were also passed through the 20 mesh sieve, added to this bag, and mixed with the other components for $2 \mathrm{~min}$. Before tablet pressing, spray dried lactose and artesunic acid were also passed through a 20 mesh sieve and mixed with excipient $\mathrm{A}$ in a zip-lock bag for $3 \mathrm{~min}$. Tablets were composed of $20 \%$ excipient A and variable amounts of artesunic acid and spray dried lactose to obtain tablets with variable properties such as hardness, amount of active ingredient, diameter, and shape. Tablets were pressed using a B2 rotary tablet press (Globe Pharma, New Brunswick, NJ) at $60 \mathrm{rpm}$. Tablet hardness was measured using a Monsanto hardness tester (Tab-Machines Ltd., Bombay, India).

\section{Desorption Electrospray Ionization Mass Spectrometry (DESI-MS)}

For quantitation purposes, all samples were treated with $25 \mu \mathrm{L}$ of $100 \mathrm{mM} \mathrm{d}_{4}$-artesunic acid IS in neat acetonitrile, unless stated otherwise. The IS solution was kept in a $-80^{\circ} \mathrm{C}$ freezer at all times, and placed on ice during use to minimize evaporation. The preferred IS spiking method consisted of addition of the IS solution by pipette deposition of a thin solution film unto the surface of the samples (Figure 1). This thin film diffuses into the sample at a rate that was observed to be dependent on the tablet porosity. Samples were allowed to air dry at room temperature before analysis. This procedure is further discussed and compared with other IS deposition approaches in the Results section.

DESI was performed using a home built DESI source [7] at an incident angle ( $\alpha)$ of $55^{\circ}$ and a collection angle $(\beta)$ of $0^{\circ}$ unless otherwise stated. Reagentless DESI was performed using a 75:25 $\mathrm{CH}_{3} \mathrm{CN}: \mathrm{H}_{2} \mathrm{O}$ mixture. This 


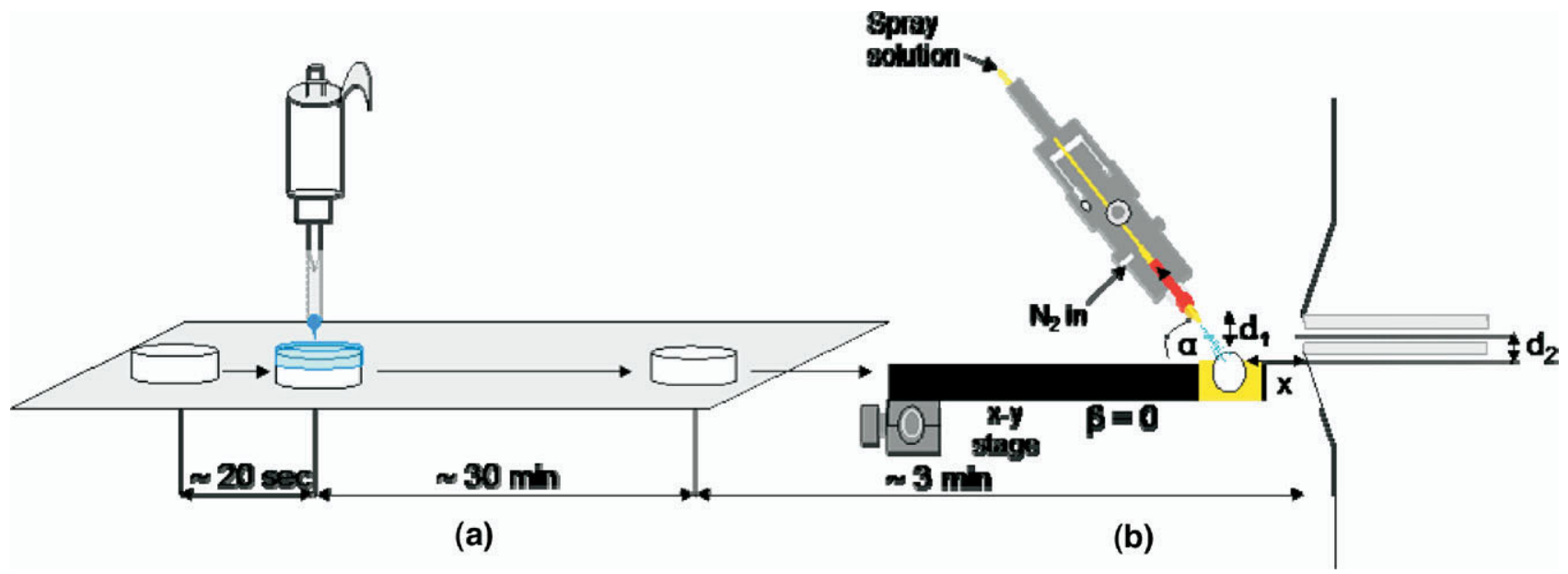

Figure 1. (a) Sample pretreatment with IS: (i) pipette deposition of a solution of $d_{4}$-artesunic acid in neat acetonitrile unto the surface of sample, (ii) drying of sample after treatment with IS, and (iii) reactive DESI analysis of air-dried samples; (b) DESI setup showing various DESI variables.

solvent mixture was doped with $100 \mu \mathrm{M}$ dodecylamine for reactive DESI experiments. Except for initial comparative experiments, all quantitative work was performed by reactive DESI. The spray solution was electrically charged with an external power supply to +3 $\mathrm{kV}$, and operated at a flow rate of $2 \mu \mathrm{L} \mathrm{min}{ }^{-1}$, unless stated otherwise. The nebulizer gas pressure was set to $110 \mathrm{psi}$, unless stated otherwise. All experiments were performed on an LCQ DECA XP+ quadrupolar ion trap mass spectrometer (Thermo Finnegan, San Jose, CA) auto-tuned for optimum detection of the ion of interest. Data were collected in full scan mode via the Xcalibur software (version 2.0, Thermo Finnegan). The ion transfer capillary was set at $300^{\circ} \mathrm{C}$ at a voltage of $14 \mathrm{~V}$ and the tube lens voltage was $-25 \mathrm{~V}$. The instrument was set to collect spectra in automatic gain mode for an ion trap injection time of $200 \mathrm{~ms}$ at 2 microscans spectrum $^{-1}$. All DESI characterization experiments were performed using genuine artesunate tablets. The assessment of the effect of sample properties on the DESI signal and the development of a calibration method were done using artesunate tablet standards with the same surface geometry as the genuine samples used for characterization experiments.

\section{Results and Discussion}

To investigate the type of ionic species formed during DESI of IS-doped genuine artesunate tablets, reagentless and reactive DESI analyses were first performed on samples treated by pipette deposition, according to the scheme depicted in Figure 1. Figure 2a shows the reagentless DESI spectrum of an IS-pretreated genuine artesunate tablet. In this mass spectrum, the analyte and IS signals are spread into various species resulting in ions at $\mathrm{m} / \mathrm{z} 407,411,791,795$, and 799 . These corresponded to sodiated analyte and IS protonated monomers and various analyte and IS protonated dimers. Various fragments could also be observed in the spec- trum as reported previously $[7,22]$. The fragmentation of analyte ions and the spread of the analyte ion signal into various channels made it complicated to choose an ion peak for quantitative analysis, as the relative intensity of the different adducts varied between experiments. Subsequent analysis of a genuine artesunate tablet doped with IS by reactive DESI showed predominantly one peak for the analyte and one for the IS with close to two orders of magnitude improvement in signal intensity compared to reagentless DESI (Figure $2 b$ ). This improvement is due to stabilization of the analyte by formation of a noncovalent complex and the consequent bunching of the analyte intensities [7]. This approach improved sensitivity and simplified the choice of analyte species for further quantitative analysis.

\section{Comparison of Internal Standard Deposition Methods}

Quantitative analysis by reactive DESI requires tablet pretreatment with an IS. Various methods were explored for this purpose including: (i) pneumatically assisted electrospray deposition, (ii) addition of IS directly into the DESI spray solution, and (iii) pipette deposition of neat acetonitrile IS solution. The performance of each of these deposition methods was assessed by evaluating the temporal stability of the signal intensity ratio of the ion at $m / z 570$ to that at $\mathrm{m} / \mathrm{z} 574$ at different spray solution flow rates and positions across the sample surface.

Nebulizer assisted electrospray deposition of IS resulted in a very unstable signal intensity ratio (Figure $\mathrm{S} 1 \mathrm{a}$, which can be found in the electronic version of this article). The ratio rises very rapidly with time, suggesting that this deposition method results in a very thin coat of IS on the sample surface, but not sufficient interdispersion with the tablet surface material. This layer is quickly depleted by the DESI jet, with increas- 

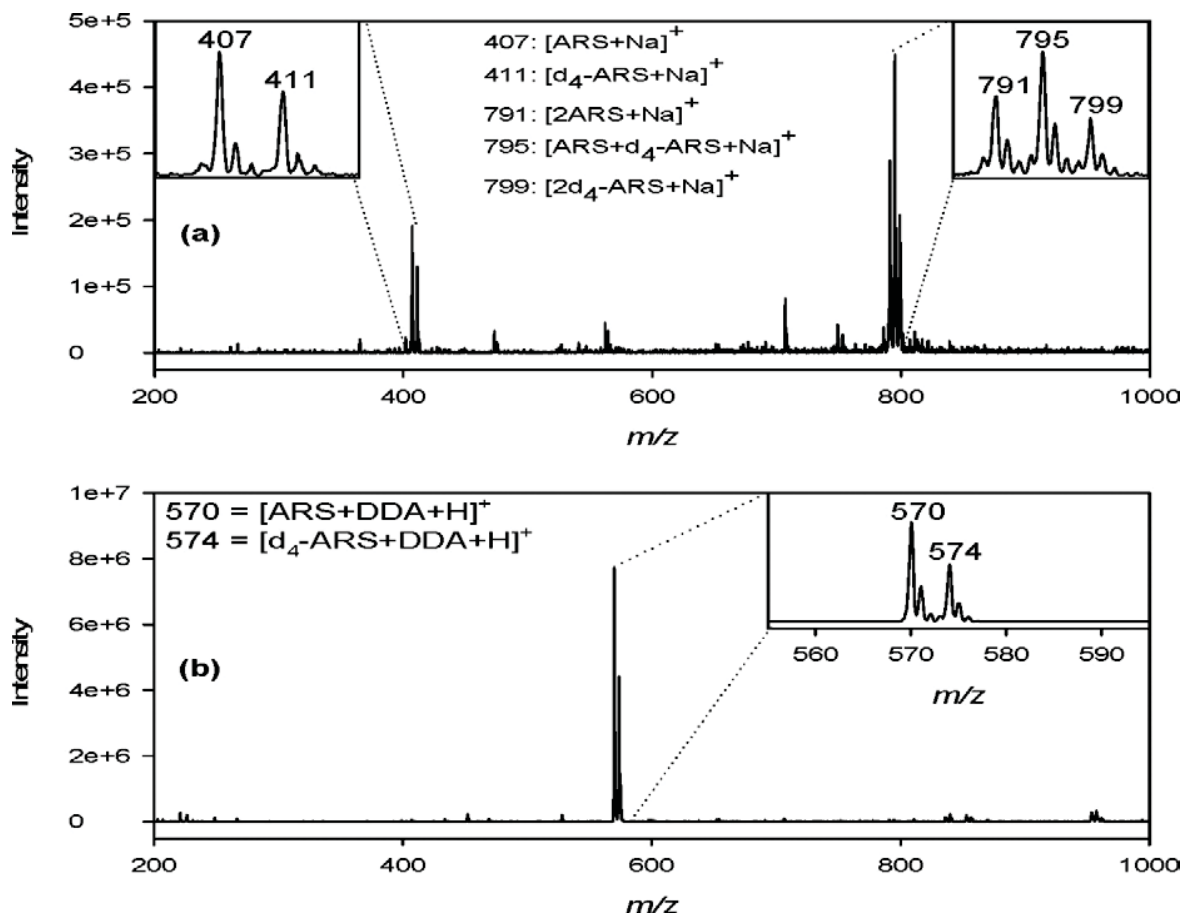

Figure 2. DESI spectra of genuine artesunate tablets pretreated with $100 \mathrm{mM}$ solution of IS after evaporation of solvent (a) reagentless DESI mode (spraying with $75: 25 \mathrm{CH}_{3} \mathrm{CN}: \mathrm{H}_{2} \mathrm{O}$ ) and (b) reactive DESI mode (spraying with $100 \mu \mathrm{M}$ dodecylamine in 75:25 $\mathrm{CH}_{3} \mathrm{CN}: \mathrm{H}_{2} \mathrm{O}$ ).

ing depletion rates at higher spray flows. To get a uniform coat of material on the sample surface, this approach required very careful control of various variables including the nebulizer gas flow rate, the spray tip-to-sample surface distance, and the elimination of any air draft currents in the room where the sample was being processed. Because of these difficulties, it was quickly realized that it would be difficult to reproduce the amount of IS deposited on different samples, which would translate into a poor day-to-day reproducibility.

Addition of a small amount of IS to the reactive DESI spray solution also resulted in an unstable signal intensity ratio (Figure S2b), with instabilities becoming more prominent at higher flow rates. Because the reactive DESI spray probes the same spot on the sample surface over a few minutes, this spot becomes progressively contaminated by the IS contained in the spray, which results in a decreasing analyte-to-IS signal ratio with time.

The method of choice adopted for these studies involved the pipette deposition of the IS dissolved in neat acetonitrile (Figure 1). After application of the IS, the acetonitrile solution flows across the sample surface, forming a homogenous liquid thin film, which is then observed to diffuse into the sample. This process requires an estimated $20 \mathrm{~s}$ for a genuine artesunate tablet. No significant disintegration of the tablets was observed during this process. After deposition, the samples were allowed to air dry at room temperature. For consistency across tablets of different hardness, $30 \mathrm{~min}$ was allowed for this step, but we believe that further optimization is possible. As several samples could be dried in parallel, this step does not necessarily decrease sample throughput significantly. Following IS deposition, four spots on the tablet surface were probed by reactive DESI, one on each tablet quadrant. Reactive DESI analysis of four surface spots required an estimated 3 min per tablet with a manual $x$-y stage.

Reactive DESI analysis of samples spiked by this deposition method at various solvent flow rates ranging from 1 to $3 \mu \mathrm{L} \mathrm{min}{ }^{-1}$ resulted in analyte and IS ionic signals which basically tracked each other (Figure 3a), resulting in a highly stable signal intensity ratio (Figure $3 b$ ). Similar analysis at various nebulizer gas pressures from 100 to 200 psi resulted in an unstable time trace when the analyte and IS signal intensities were summed (Figure 4a), but produced a stable signal intensity ratio at all nebulizer gas flow rates investigated (Figure $4 \mathrm{~b}$ ). The intensity ratio was also found to be largely stable for different positions across the sample surface, with a relative standard deviation of $7.9 \%(n=5)$ which suggests a homogenous distribution of IS over the surface of the sample. This ratio was also largely stable for a wide range of DESI spray flow rate and nebulizer gas pressures, with relative standard deviations of $5.1 \%(n=7)$ and $5.0 \%(n=6)$, respectively, as shown in Table 1 . Depth profiling of a genuine artesunate sample treated with 25 $\mu \mathrm{L}, 100 \mathrm{mM}$ IS in neat acetonitrile following the pipette deposition method showed a stable analyte-to-IS signal intensity ratio when the same spot on the sample was interrogated with the reactive DESI jet for to $60 \mathrm{~min}$, 
(a)

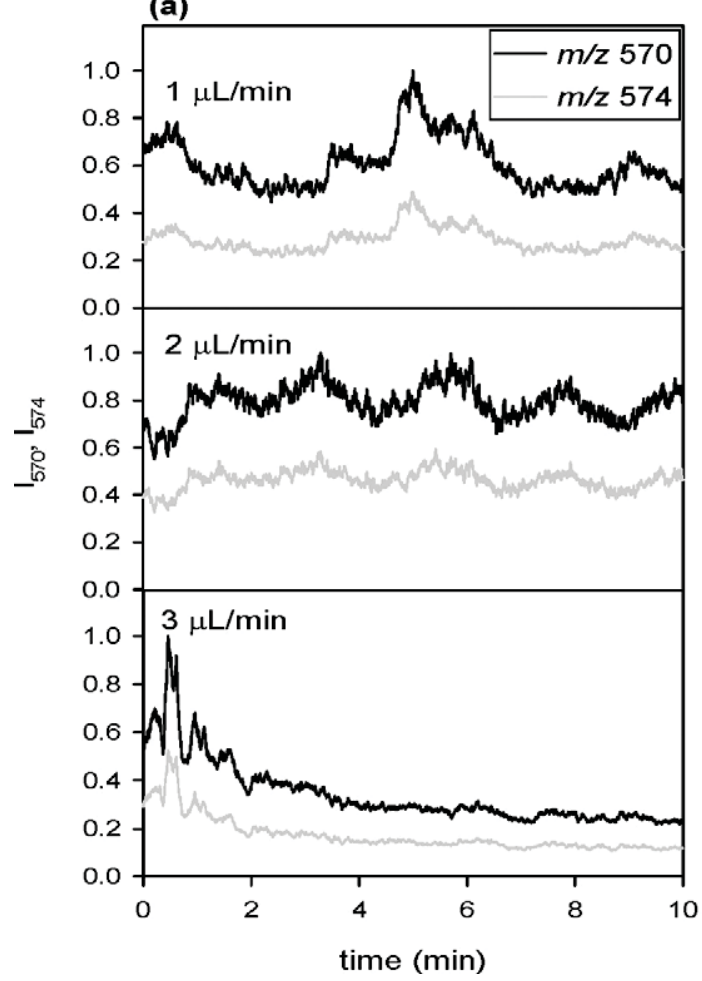

(b)

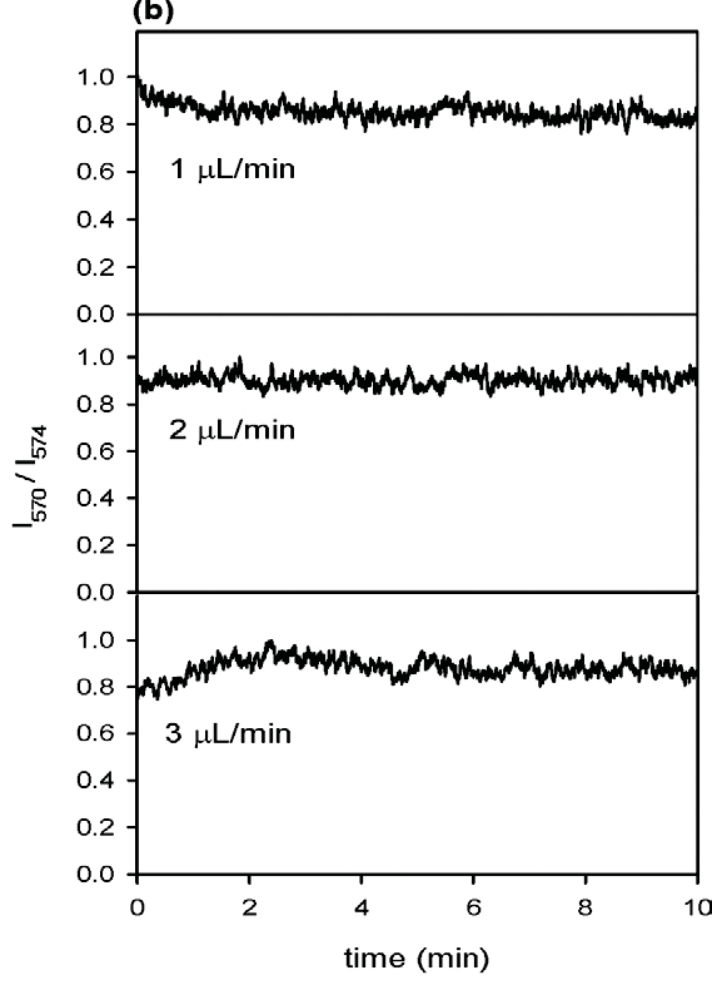

Figure 3. Normalized ion intensity traces at various solution flow rates by reactive DESI. (a) Relative intensity of ions at $\mathrm{m} / \mathrm{z} 570$ and $\mathrm{m} / \mathrm{z} 574$, (b) intensity ratio of the $\mathrm{m} / \mathrm{z} 570$ ion to the ion at $\mathrm{m} / \mathrm{z} 574$. Tablets were pretreated with $25 \mu \mathrm{L}$ of $100 \mathrm{mM}$ IS before analysis.

forming a crater of $\sim 0.3 \mathrm{~mm}$ in depth (Figure S2). This suggests a minimum penetration of the IS up to this depth. All subsequent experiments were performed by

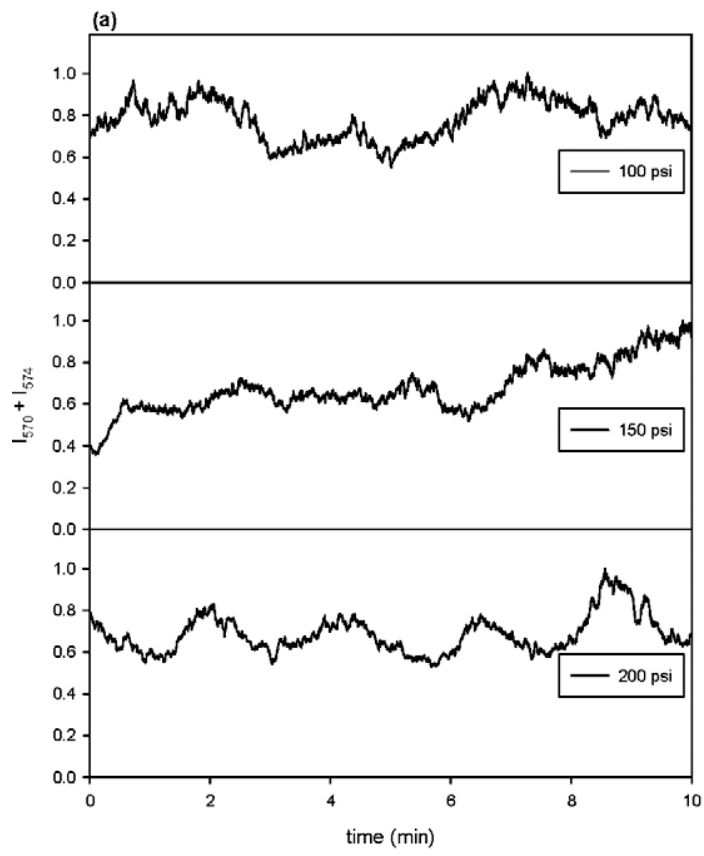

this IS deposition method at a DESI spray solution flow rate of $2 \mu \mathrm{L} \mathrm{min}{ }^{-1}$ and a nebulizer gas pressure of 110 psi.

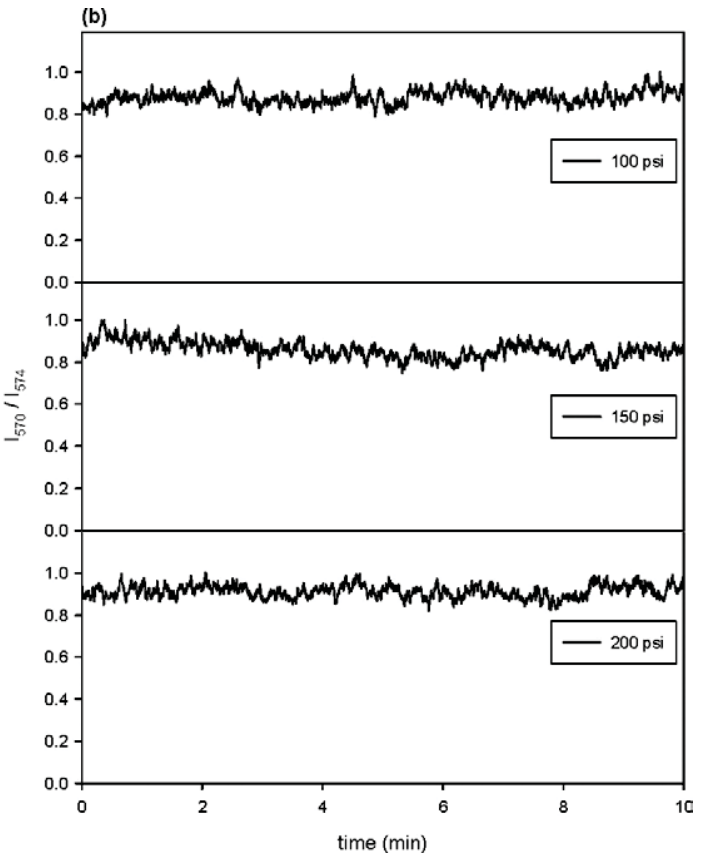

Figure 4. Normalized ion intensity traces at various nebulizer gas pressures by reactive DESI. (a) Total ion intensity generated from the ions and $\mathrm{m} / \mathrm{z} 570$ and $\mathrm{m} / \mathrm{z} 574$ (b) Intensity ratio of the ion at $m / z 570$ to the ion at $\mathrm{m} / \mathrm{z} 574$. Tablets were pretreated with $25 \mu \mathrm{L}$ of $100 \mathrm{mM}$ IS before analysis. 
Table 1. Comparison of the reproducibility of the absolute DESI signal versus the signal intensity ratio of analyte-to-IS for various DESI variables

\begin{tabular}{|c|c|c|}
\hline \multirow[b]{2}{*}{ DESI variable } & \multicolumn{2}{|c|}{ RSD (\%) } \\
\hline & $I_{570}+I_{574}$ & $\mathrm{I}_{570} / \mathrm{I}_{574}$ \\
\hline Solution flow rate $\left(1-7 \mu \mathrm{L} \mathrm{\operatorname {min } ^ { - 1 } )}\right.$ & 29.9 & 5.1 \\
\hline Nebulizer gas pressure (50-300 psi) & 83.4 & 5.0 \\
\hline Tip-to-surface distance, $\mathrm{d}_{1}(1.2-6.1 \mathrm{~mm})$ & 33.6 & 10.6 \\
\hline Spectrometer orifice-to-surface distance, $\mathrm{d}_{2}(0.8-1.4 \mathrm{~mm})$ & 46.4 & 7.8 \\
\hline Sample-to-spectrometer orifice distance, $\times(2.2-7.3 \mathrm{~mm})$ & 66.8 & 8.8 \\
\hline Spray incident angle, $\alpha\left(40^{\circ} 90^{\circ}\right)$ & 82.1 & 11.4 \\
\hline Collection angle, $\beta\left(0^{\circ}-30^{\circ}\right)$ & 72.6 & 6.4 \\
\hline
\end{tabular}

Effect of DESI Setup Geometry on the Analyte-to-Internal Standard Signal Intensity Ratio

While DESI spray conditions can be fixed, it is difficult to exert strict reproducible control on all the geometric settings over long periods of time. Takáts et al. have suggested that one possible solution is to perform the analysis in the presence of an IS [18]. In this trend, the dependence of the DESI signal on the various geometric parameters describing the DESI setup, including the DESI spray tip-to-surface distance $\left(\mathrm{d}_{1}\right)$, sample-to-spectrometer orifice distance $(\mathrm{x})$, spectrometer inlet-to-surface distance $\left(\mathrm{d}_{2}\right)$, spray incidence angle $(\alpha)$, and droplet collection angle $(\beta)$, was investigated.

Summarized in Table 1 are results of the effect of the above-mentioned DESI variables on the analyte-to-IS signal intensity ratio in comparison to the absolute signal, as stated by their relative standard deviations. For the variables $\alpha$ and $\beta$, the relative standard deviations observed for the normalized signal intensity ratio were $11.4 \%(n=6)$ and $6.4 \%(n=7)$, as opposed to $82.1 \%$ and $72.6 \%$, respectively, when the absolute signals were monitored. For the variables $d_{1}, d_{2}$, and $x$, the observed relative standard deviations were 10.6\% $(n=6)$, $7.8 \%(n=4)$, and $8.8 \%(n=6)$ for the signal intensity ratio as opposed to $33.6 \%, 46.4 \%$ and $66.8 \%$, respectively, for the absolute signal. These results show lower relative standard deviations when the analyte-to-IS signal intensity ratio was monitored if compared to the sum of the absolute signals corresponding to the analyte and IS. This indicates a significant independence of the analyte-to-IS intensity ratio for all DESI spray condition and geometrical parameters, and suggests the potential of this approach for the direct quantitative analysis of active ingredients in solid pharmaceutical tablets.

\section{Effect of Sample Properties on the Analyte-to-Internal Standard Intensity Ratio}

In our previous screening experiments of potentially counterfeit artesunate samples collected in the field, we observed a significant dependence of the DESI signal on tablet hardness [7]. Is it thus important to understand the effect of this and other sample-related variables on the analyte-to-IS signal intensity ratio, so as to establish controlled conditions under which robust quantitative results can be obtained.

Figure 5 shows the effect of sample hardness on the analyte-to-IS signal intensity ratio for artesunate tablet standards containing three different amounts of the active ingredient. All samples were treated with identical amounts of IS and analyzed by reactive DESI at a spray solution flow rate of $2 \mu \mathrm{L} \mathrm{min}{ }^{-1}$. An almost linear decrease in signal intensity ratio was observed for hardness values below $5 \mathrm{~kg}$. For harder tablets, the signal ratio becomes relatively constant, depending primarily on the analyte concentration. As the IS solution percolates into the sample at a rate that depends on sample porosity, which increases with decreasing sample hardness, the IS percolates deeper and faster into the body of softer samples, with the end result of a more "dilute" superficial IS zone. This results in a larger $\mathrm{m} / \mathrm{z} 570$ to 574 signal intensity ratio during reactive DESI analysis. For samples prepared with hardness values larger than 5 $\mathrm{kg}$, the results shown in Figure 5 suggest a quasiconstant degree of percolation of the IS into the tablet body. In this case, the rate of evaporative loss of the

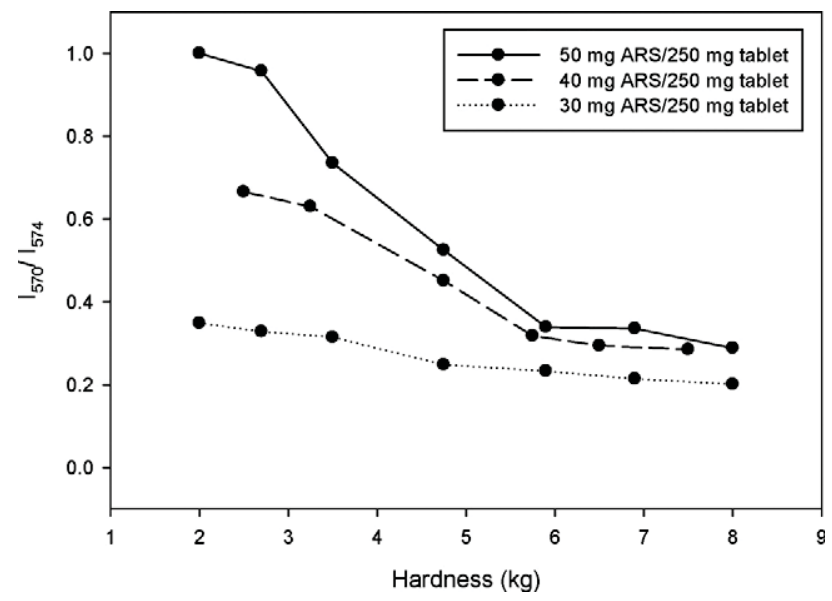

Figure 5. Dependence of the $m / z 570$ to 574 ion intensity ratio for reactive DESI analysis of artesunate tablet standards with varying hardness. The intensity ratios were normalized to that of the maximum value. Tablets were pretreated with $25 \mu \mathrm{L}$ of $100 \mathrm{mM}$ IS and analyzed by reactive DESI at a solution flow rate of $2 \mu \mathrm{L}$ $\min ^{-1}$. 
IS solution solvent is larger than the percolation rate, determining the amount of material migrating into the tablet surface.

To further examine the distribution of the IS into the tablet sample, depth profiling analysis was performed at two different spray solvent rates $(2$ and 6 $\mu \mathrm{L} \min ^{-1}$ ) for samples prepared at three different hardness values $(2.7,5.9$, and $8.0 \mathrm{~kg}$, Figure S3). At 2 $\mu \mathrm{L} \min ^{-1}$ the analyte-to-IS signal ratio was stable, with relative standard deviations below $10 \%$ for all hardness values investigated, indicating a homogenous distribution of the IS within the outermost tablet layers probed at this flow rate. Significant disintegration of the sample surface was not observed. However, if the flow rate was increased to $6 \mu \mathrm{L} \mathrm{min}^{-1}$, the analyte-to-IS signal ratio was observed to gradually increase over time. This validates our hypothesis that a gradient of the IS is established within the tablet surface. Tablets with different hardness showed a different temporal evolution of the IS-to-analyte signal ratio due to differences in the rate at which the spray ablated the surface. To ensure optimum dissolution properties, optimum dosage, and absorption, pharmaceutical tablets are usually prepared with hardness lower than $5 \mathrm{~kg}$, meaning that the relative hardness of samples and standard must be used as a correction factor for performing quantitative DESI.

Quantitative analysis via pipette deposition of an IS solution requires that all samples be treated with the same amount of IS per unit tablet area. It was thus also necessary to evaluate the change in analyte-to-IS signal intensity ratio as a function of the surface area treated with IS. Results observed from the reactive DESI analysis of duplicate tablets at two different diameters (9 and $11 \mathrm{~mm}$ ) treated with identical amount of IS indicate that the analyte-to-IS signal intensity ratio increases with increasing tablet diameter. The average intensity ratios of analyte-to-IS observed for $9 \mathrm{~mm}$ and $11 \mathrm{~mm}$ diameter, 250-mg tablets containing $50 \mathrm{mg}$ artesunate were 4.5 and 7.7, respectively. Because in both cases the analyte solution is completely dispersed over the whole tablet surface, and the tablet thickness was kept constant, a lower amount of IS percolated per unit surface area when the tablet diameter was increased.

In addition to tablet diameter, the effect of tablet shape on the analyte-to-IS signal intensity ratio was investigated. Standard tablet samples with flat and convex topology treated with identical amounts of IS solution showed a negligible change in analyte-to-IS signal intensity ratio with convex tablets showing slightly higher ratios compared with flat ones. The observed average percentage increase in the intensity ratio was $9.9 \%$. This is probably due to the larger surface area of the convex sample, which results in a lower amount of IS per surface area, and thus a higher analyte-to-IS signal intensity ratio.

\section{Calibration and Direct Quantitation of Artesunate in Pharmaceutical Samples by Reactive DESI}

The large independence of the analyte-to-IS signal intensity ratio observed for all DESI variables promises applicability of reactive DESI for direct quantitative screening. As a first step in establishing the quantitative capabilities of the approach proposed here, a calibration curve for the prediction of unknown samples was generated using 250-mg tablet standards with varying artesunate amounts, prepared at a hardness close to that of genuine samples $(2.4 \pm 0.2 \mathrm{~kg})$, with a flat surface shape, and with diameters of $9 \mathrm{~mm}$ (area $=63.6$ $\mathrm{mm}^{2}$ ). Three replicate tablets were prepared at each level of the active ingredient in the range of 0.02 to 0.32 $\mathrm{mg}$ artesunate $\mathrm{mg}^{-1}$ tablet. The calibration curve showed good linearity over the investigated concentration range with a correlation coefficient of 0.9985 . The limit of detection defined as the analyte concentration giving a signal equal to the blank signal plus three standard deviations was calculated from the calibration curve to be equal to $0.018 \mathrm{mg}$ artesunate $\mathrm{mg}^{-1}$ tablet ( $4.5 \mathrm{mg} / 250 \mathrm{mg}$ tablet). A set of test samples, with amounts of artesunate that fell in the calibration curve range, was prepared for validation purposes. Results showed a good correspondence between the expected and the predicted amounts of artesunate present in the validation sample set, with a correlation coefficient of 0.9971 , and $y$-intercept and slope values of $0.0109 \pm$ 0.0197 and $1.139 \pm 0.108$, respectively. These results validate the applicability of this quantitative DESI MS approach to samples with known (or constant) hardness, surface area, and shape, such as those encountered in a quality control laboratory setting.

A more challenging application of this DESI approach is the quantitation of active ingredients in samples with hardness not exactly matched to the calibration standards. This would be the case, for example, of drugs suspected of being counterfeit, which are collected in the field for further off-field forensic analysis. As a first test of the performance of the proposed method in this scenario, three different artesunate tablet samples from Mekophar pharmaceutical collected in the field with a measured hardness of $2.7 \pm 0.2 \mathrm{~kg}$ and diameter of $10.1 \mathrm{~mm}$ were treated with IS solution and analyzed by reactive DESI against a calibration curve created with standard tablets with $2.4 \pm 0.2 \mathrm{~kg}$ hardness and $9 \mathrm{~mm}$ diameter. Table 2 summarizes the results of

Table 2. Quantitation of artesunate in genuine sample by DESI MS compared with the expected amount present in the sample determined by HPLC with spectrophotometric detection

\begin{tabular}{lccc}
\hline & \multicolumn{3}{c}{ Artesunate $\left(\mathrm{mg} \mathrm{tablet}^{-1}\right)$} \\
\cline { 2 - 4 } Sample & $\begin{array}{c}\text { Predicted with no } \\
\text { correction }\end{array}$ & $\begin{array}{c}\text { Predicted with } \\
\text { correction }\end{array}$ & HPLC \\
\hline \hline Tablet 1 & $73 \pm 4$ & $58 \pm 3$ & $53 \pm 2$ \\
Tablet 2 & $73 \pm 5$ & $58 \pm 4$ & $56 \pm 2$ \\
Tablet 3 & $68 \pm 4$ & $53 \pm 3$ & $50 \pm 2$ \\
\hline
\end{tabular}


these analyses. An average relative standard deviation of $6 \%$ was obtained for the predicted amount of artesunate present in the three samples, which compares relatively well with the $2 \%$ observed for HPLC analysis. Comparison of the predicted amount of artesunate to the amount determined by HPLC showed significant deviations ( $35 \%$ on average) if uncorrected by tablet hardness and surface area. Upon normalization of the analyte-to-IS signal ratio by the tablet hardness and surface area, and comparison to the standard containing $50 \mathrm{mg}$ artesunate/tablet, the difference between the artesunate amount determined by DESI and the amount determined by HPLC was reduced to $6 \%$ on average. This is a clear indication that the sample physical properties play a critical role in quantitative analysis by DESI. When corrected, the average accuracy obtained by reactive DESI quantitation can be considered sufficient for determining active ingredient content with an acceptable degree of accuracy (94\%) for a highthroughput pharmaceutical screening assay such as this.

\section{Conclusions}

Reactive DESI has been shown to be successful as a high-throughput quantitative approach for the direct screening of antimalarial tablets. The controlled deposition of an internal standard makes it possible to perform quantitative measurements independently from the DESI setup variables. The method shows a precision of $6 \%(n=4)$ and an accuracy of $94 \%$, which is largely acceptable for the rapid quantitation of active ingredients in pharmaceutical tablets. Sample hardness and surface area are important factors to be taken into account when implementing this method. Although correction schemes to take into account tablet hardness and shape are feasible, it is preferable that, whenever possible, the calibration sample set has the same characteristics as the unknowns. Future work will be geared towards investigating the role of charge competition effects in DESI, in an effort towards quantifying multiple components without chromatographic separation.

\section{Acknowledgments}

This study was supported by a graduate scholarship from the U.S. Pharmacopeia to LN, an NSF CAREER Award to FMF, and by WPRO/WHO. The collection of artesunate samples was funded by the Wellcome Trust of Great Britain as part of the Wellcome Trust-South East Asian Oxford Tropical Medicine Research Collaborations. The authors are extremely grateful to all who have assisted in the collection of samples. We are also extremely grateful to Dr. Paul Newton for this collaboration.

\section{References}

1. Aebersold, R.; Goodlett, D. R. Mass Spectrometry in Proteomics. Chem. Rev. 2001, 101, 269-295.

2. Griffin, T. J.; Goodlett, D. R.; Aebersold, R. Advances in Proteome Analysis by Mass Spectrometry. Curr. Opin. Biotechnol. 2001, 12, 607612.

3. Chace, D. H. Mass Spectrometry in the Clinical Laboratory. Chem. Rev. 2001, 101, 445-477.

4. Frahm, J. L.; Howard, B. E.; Heber, S.; Muddiman, D. C. Accessible Proteomics Space and Its Implications for Peak Capacity for Zero-, One-, and Two-Dimensional Separations Coupled with FT-ICR and TOF Mass Spectrometry. J. Mass Spectrom. 2006, 41, 281-288.

5. Takats, Z.; Wiseman, J. M.; Gologan, B.; Cooks, R. G. Mass Spectrometry Sampling under Ambient Conditions with Desorption Electrospray lonization. Science 2004, 306, 471-473.

6. Cody, R.; Laramee, J.; Durst, H. Versatile New Ion Source for the Analysis of Materials in Open Air under Ambient Conditions. Anal. Chem. 2005, 77, 2297-2302.

7. Nyadong, L.; Green, M.; De Jesus, V.; Newton, P. N.; Fernandez, F. M. Reactive Desorption Electrospray Ionization Linear Ion Trap Mass Spectrometry of Latest-Generation Counterfeit Antimalarials Via Noncovalent Complex Formation. Anal. Chem. 2007, 79, 2150-2157.

8. Cotte-Rodriguez, I.; Chen, H.; Cooks, R. G. Rapid Trace Detection of Triacetone Triperoxide (TATP) by Complexation Reactions During Desorption Electrospray Ionization. Chem. Commun. 2006, 953-955.

9. Cotte-Rodriguez, I.; Takats, Z.; Talaty, N.; Chen, H.; Cooks, R. G. Desorption Electrospray Ionization of Explosives on Surfaces: Sensitivity and Selectivity Enhancement by Reactive Desorption Electrospray Ionization. Anal. Chem. 2005, 77, 6755-6764.

10. Chen, H.; Talaty, N. N.; Takats, Z.; Cooks, R. G. Desorption Electrospray Ionization Mass Spectrometry for High-Throughput Analysis of Pharmaceutical Samples in the Ambient Environment. Anal. Chem. 2005, 77, 6915-6927.

11. Leuthold, L. A.; Mandscheff, J. F.; Fathi, M.; Giroud, C.; Augsburger, M.; Varesio, E.; Hopfgartner, G. Desorption Electrospray Ionization Mass Spectrometry: Direct Toxicological Screening and Analysis of Illicit Ecstasy Tablets. Rapid Commun. Mass Spectrom. 2006, 20, 103-110.

12. Ricci, C.; Nyadong, L.; Fernandez, F. M.; Newton, P. N.; Kazarian, S. Combined Fourier Transform Infrared Imaging and Desorption Electrospray Ionization Linear Ion Trap Mass Spectrometry for the Analysis of Counterfeit Antimalarial Tablets. Anal. Bioanal. Chem. 2007, 387, 551-559.

13. Weston, D. J.; Bateman, R.; Wilson, I. D.; Wood, T. R.; Creaser, C. S Direct Analysis of Pharmaceutical Drug Formulations Using Ion Mobility Spectrometry/Quadrupole-Time-of-Flight Mass Spectrometry Combined with Desorption Electrospray Ionization. Anal. Chem. 2005, 77, 7572-7580.

14. Williams, J. P.; Lock, R.; Patel, V. J.; Scrivens, J. H. Polarity Switching Accurate Mass Measurement of Pharmaceutical Samples Using Desorption Electrospray Ionization and a Dual Ion Source Interfaced to an Orthogonal Acceleration Time-of-Flight Mass Spectrometer. Anal. Chem. 2006, 78, 7440-7445.

15. Williams, J. P. Patel, V. J.; Holland, R. Scrivens, J. H. The Use of Recently Described Ionization Techniques for the Rapid Analysis of Some Common Drugs and Samples of Biological Origin. Rapid Commun. Mass Spectrom. 2006, 20, 1447-1456.

16. Williams, J. P.; Scrivens, J. H. Rapid Accurate Mass Desorption Electrospray Ionization Tandem Mass Spectrometry of Pharmaceutical Samples. Rapid Commun. Mass Spectrom. 2005, 19, 3643-3650.

17. Venter, A.; Cooks, R. G. Desorption Electrospray Ionization in a Small Pressure-Tight Enclosure. Anal. Chem. 2007, 79, 6398-6403.

18. Takáts, Z.; Wiseman, J. M.; Cooks, R. G. Ambient Mass Spectrometry Using Desorption Electrospray Ionization (DESI): Instrumentation, Mechanisms, and Applications in Forensics, Chemistry, and Biology. J. Mass Spectrom. 2005, 40, 1261-1275

19. Van Berkel G, J. T.; B. A.; Kertesz, V. Thin-Layer Chromatography/ Desorption Electrospray Ionization Mass Spectrometry: Investigation of Goldenseal Alkaloids. Anal. Chem. 2007, 79, 2778-2789.

20. International Medicine Product Anti-Counterfeiting Taskforce (IMPACT). http://www.who.int/medicines/services/counterfeit/ impact/TheNewEstimatesCounterfeit.pdf, 2006.

21. Newton, P. N.; Green, M.; Fernandez, F. M.; Day, N. P. J.; White, N. J. Counterfeit Anti-Infective Drugs. Lancet Infect. Dis. 2006, 6, 602-612.

22. Fernandez, F. M.; Cody, R. B.; Green, M.; Hampton, C. Y.; McGready, R.; Sengaloundeth, S.; White, N. J.; Newton, P. N. Characterization of Solid Counterfeit Drug Samples by Desorption Electrospray Ionization and Direct-Analysis-in-Real-Time Coupled to Time-of-Flight Mass Spectrometry. Chem. Med. Chem. 2006, 1, 702-705.

23. Quingaosu Antimalaria Coordinating Research Group. J. Traditional Chinese Med. 1982, 2, 9-16.

24. Singh, B. R.; Prakash, K. A.; Tarun, S.; Singh, K. S. P. Patent No. PCT/IB2002/005063, 2004. 